\title{
MODELO PARA A DETERMINAÇÃO DO ESPAÇAMENTO ENTRE DESAGUADOUROS EM ESTRADAS NÃO PAVIME NTADAS ${ }^{(\mathbf{1})}$
}

\author{
Nori Paulo Griebeler ${ }^{(2)}$, Fernando Falco Pruski ${ }^{(3)}$, J osé Márcio Alves da Silva(4), \\ Márcio Mota Ramos ${ }^{(5)}$ \& Demetrius David da Silva(6)
}

\begin{abstract}
RESUMO
As estradas não pavimentadas são de fundamental importância para o desenvolvimento social e econômico do Brasil, sendo a erosão provocada pela água no leito e nas margens destas estradas um dos principais fatores para sua degradação. Neste artigo, apresenta-se um novo modelo para a determinação do espaçamento entre desaguadouros em estradas não pavimentadas e, conseqüentemente, para a redução dos problemas associados à erosão hídrica. Utilizando o modelo de ondas cinemáticas, determina-se o hidrograma de escoamento superficial no canal de drenagem da estrada e, a partir deste, é calculada a tensão provocada pelo escoamento. Sempre que a tensão cisal hante causada pelo escoamento supera a tensão crítica para cisalhamento do solo ocorre o seu desprendimento. A diferença entre essas tensões ci sal hantes é multi plicada pela erodibilidade do solo e pela área em que ocorre a aplicação da tensão cisalhante para estimar a perda de solo provocada pelo escoamento. 0 espaçamento máximo entre desaguadouros é obtido, comparando-se a perda de solo provocada pelo escoamento com a perda de solo tolerável. O comprimento máximo do canal é aquele para o qual a perda tolerável é atingida. 0 modelo permite determinar o espaçamento entre desaguadouros considerando as características das áreas que contribuem para o escoamento e a resistência do solo. As simulações realizadas mostraram que o modelo é sensível às alterações na erodibilidade e na tensão crítica de cisalhamento do solo, bem como à declividade do canal, permitindo ainda que, por meio da alteração no aprofundamento tolerável para o canal, os espaçamentos possam ser ampliados ou reduzidos.
\end{abstract}

Termos de indexação: conservação do solo, modelo hidrológico, tensão cisalhante, erosão hídrica.

\footnotetext{
(1) Trabalho extraído de Tese de Doutorado, do primeiro autor. Recebido para publicação em abril de 2003 e aprovado em março de 2005.

(2) Professor Adjunto da Universidade Federal de Goiás - UFG. Caixa Postal 131, CEP 74001-970 Goiânia (GO). E-mail: griebeler@yahoo.com.br

(3) Professor Titular do Departamento de Engenharia Agrícola, Universidade Federal de Viçosa - UFV. Campus Universitário, CEP 36570-000 Viçosa (MG). Bolsista do CNPq. E-mail: ffpruski@ufv.br

(4) Engenheiro Civil, DS, Departamento de Engenharia Agrícola, UFV. E-mail: jmarcio@ufv.br

(5) Professor Titular do Departamento de Engenharia Agrícola, UFV. Bolsista do CNPq. E-mail: mmramos@ufv.br

(6) Professor Adjunto do Departamento de Engenharia Agrícola, UFV. Bolsista do CNPq. E-mail: dds@ufv.br
} 


\title{
SUMMARY: A MODEL TO DETERMINE THE SPACING BETWEEN WATER OUTLETS IN UNPAVED ROADS
}

\begin{abstract}
Unpaved roads areessential for theeconomic and social devel opment in Brazil. Water erosion is one of the key factors causing their degradation. This study developed a new model of determination of spacing between water outlets in unpaved roads. The surface runoff hydrograph in theroad channel was estimated using the cinematic wavemodel and the runoff shear stress was calculated using the flow rate obtained in the hydrograph. Detachment occurs when theflow shear stress exceeds thecritical soil shear stress. The soil loss was determi ned multiplying the differencebetween theflow shear stress and thecritical soil shear stress by thesoil erodibility and thearea wheretherunoff shear stress is appl ied. Themaximum spacing between thewater outlets is calculated by the comparison of the soil loss produced by runoff with the tolerable soil loss. The model determines the road outlet spacing considering thefeatures of theareas contributing to therun off and thesoil resistance. Thesimulations showed that themodel is sensitiveto changes in thesoil erodibility, critical soil shear stress and channe slope, including the possibility of increasing or reducing the spacing by altering the tolerablechannel depth.
\end{abstract}

Index terms: soil conservation, hydrologic model, shear stress, water erosion.

\section{NTRODUÇÃO}

O Brasil possui aproximadamente $1.725 .000 \mathrm{~km}$ de estradas distribuídas nas diferentes regiões, das quais mais de 90 \% não são pavimentadas (DNER, 2000).

Os custos para construção e manutenção das estradas são bastante el evados, devendo sua construção ser realizada de maneira a considerar todos os fatores que possam vir a prejudicar a sua estrutura. Neste sentido, a anál ise deve ser bastante criteriosa, uma vez que, para estradas não pavimentadas, o material doleito apresenta grande variabilidade, sendo, normalmente, obtido no próprio local de construção da estrada.

A erosão provocada pela água no leito e nas margens das estradas é um dos principais fatores para sua degradação, sendo responsável por mais da metade das perdas de sol o no Estado de São Paulo (Anjos Filho, 1998). No estado da Carolina do Norte, Estados Unidos, Grace III et al. (1998) observaram que mais de $90 \%$ do sedimento produzido em áreas florestais advém das estradas, sendo a drenagem inadequada o principal fator responsável. A maior porção do sedimento produzido na superfície da estrada é de tamanho inferior a $2 \mathrm{~mm}$, o qual, de acordocom Reid \& Dunne (1984), éo mais prejudicial aos recursos hídricos, apresentando o agravante de que o material erodido das estradas move-se, comumente, diretamente dos canais de drenagem aos cursos d'água.

Mesmo em estradas de pequeno porte, local izadas nas áreas internas de propriedades rurais, destinadas apenas ao uso particular, podem ocorrer problemas erosivos, podendo tanto a estrada ser prejudicada pela ocorrência de erosão e aporte de sedimentos advindos das áreas marginais, como ser a responsável pela erosão nestas áreas (Nogami \& Villibor, 1995).

A redução dos problemas de erosão nas estradas deterra pode ser obtida pela adoção de medidas que evitem que a água proveniente do escoamento superficial, tanto aquel e gerado na própria estrada como o proveniente das áreas às suas margens, acumule-se na estrada e passe a utilizá-la para o seu escoamento. A água escoada pela estrada deve ser coletada nas suas laterais e encaminhada, de modo controlado, para os escoadouros naturais, artificiais, bacias de acumulação ou outro sistema de retenção localizado no terreno marginal.

Diversos autores (Bublitz \& Campos, 1992; Bertolini et al., 1993; Pastore, 1997) têm recomendado a adoção de espaçamentos entre desaguadouros ou bacias de acumulação de água baseados apenas na declividade do terreno e na textura do solo. Os valores recomendados são muito genéricos e não levam em consideração a vazão de escoamento a ser transportada pel os canais, a condição hidráulica em que estes canais se encontram, bem como a resistência que os diferentes tipos de solos apresentam ao desprendimento. O conhecimento de todos estes fatores é necessário para o adequado dimensionamento dos sistemas de drenagem.

De acordo com Nogami \& Villibor (1995), a erodibilidade do solo é um dos fatores mais importantes para a escolha do projeto mais apropriado para controlar a erosão ao longo das rodovias. Outro fator de grande importância para determinação da capacidade resistível do solo à erosão é a tensão crítica de cisalhamento, a qual correspondeà máxima tensão que pode ser aplicada ao sol o sem que haja remoção de suas partículas. 
Tendo em vista a carência e a conseqüente necessidade de métodos para a implementação de técnicas mais seguras para o dimensionamento de sistemas de drenagem em estradas de terra, elaborou-se o presente trabalho, que teve como objetivo o desenvolvimento de um modelo para a determinação do espaçamento entre desaguadouros em estradas não pavimentadas.

\section{MATERIAL E MÉTODOS}

Com vistas em obter o espaçamento entre desaguadouros, foram determinadas as condi ções de escoamento no canal de drenagem da estrada, comparando-as com a capacidade que o solo apresenta de resistir ao desprendimento de partículas pelo escoamento superficial. A partir de então efetuou-se a quantificação da perda de solo no canal, que foi comparada a um limite de perdas tolerável.

\section{Determi nação das condições de escoamento no canal da estrada}

Para determinar a variação da vazão ao longo do tempo (hidrograma) no canal da estrada, determinaram-se, inicialmente, os hidrogramas advindos das áreas de contribuição, sendo considerados no model otanto o leito da estrada como a área externa ou talude.

\section{Determinação do hidrograma de escoamento superficial pelo modelo}

Para determinar o hidrograma de escoamento, as áreas de contribuição, foram divididas em linhas e colunas, formando uma mal ha regular sobre o terreno (Figura 1). Nesta figura, observa-sequeas linhas são tomadas no sentido transversal, e as colunas no sentido longitudinal à estrada.

Para determinar o hidrograma, foi utilizado o método desenvolvido por Silva (1999), no qual o escoamento superficial é avaliado para cada célula da malha e somado ao escoamento da célula seguinte, sendo o hidrograma de escoamento, para ambas as áreas de contribuição, determinado para cada linha, na última coluna, junto ao canal .

Como as características de escoamento são diferentes no leito da estrada e na área externa, os tempos para que cada hidrograma atinja o canal também são diferentes.

O hidrograma no canal é obtido acumulando os hidrogramas correspondentes à contribuição de cada linha, sendo somadas as vazões referentes ao leito da estrada e à área de contribuição externa a esta, obedecendo à coincidência dos tempos de chegada do escoamento ao canal.

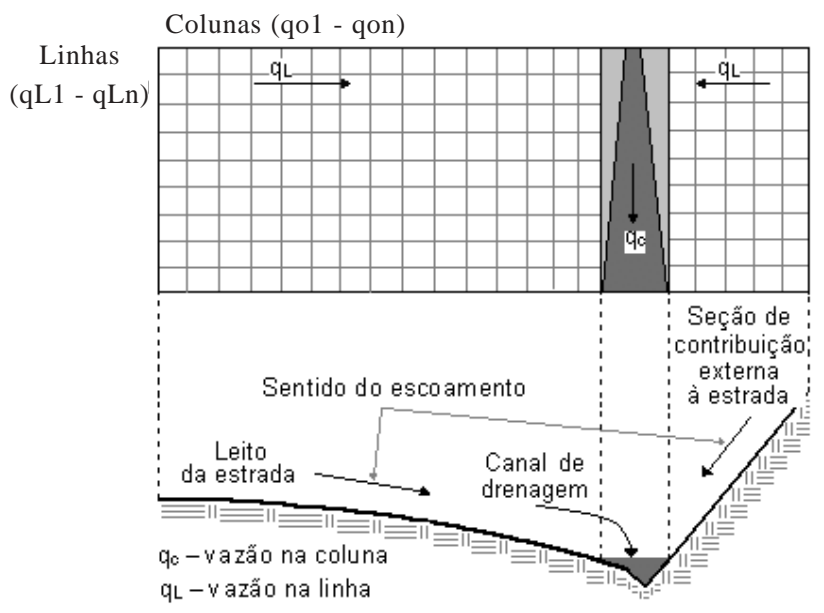

Figura 1. Representação esquemática da divisão do leito da estrada e da área externa de contribuição na forma de malha para a determinação do hidrograma de escoamento superficial no canal de drenagem da estrada.

As características das áreas de contribuição utilizadas pelo modelo para a obtenção do hidrograma são: a largura, o comprimento, a declividade, a taxa de infiltração estável de água no solo, a rugosidade hidráulica e o tipo de cobertura vegetal.

\section{Modelagem do escoamento superficial advindo das áreas de contribuição}

O model o utiliza o método das ondas cinemáticas, conforme expresso por J ulien et al. (1995) e MuñozCarrera \& Parsons (1999).

$$
\frac{\partial \mathrm{h}}{\partial \mathrm{t}}+\frac{\partial \mathrm{q}}{\partial \mathrm{x}}=\mathrm{i}_{\mathrm{i}}-\mathrm{T}_{\mathrm{i}}
$$

em que

$$
\begin{aligned}
& \mathrm{h}=\text { profundidade do escoamento }(\mathrm{m}), \\
& \mathrm{t}=\text { tempo }(\mathrm{s}), \\
& \mathrm{q}=\text { vazão por unidade de largura }\left(\mathrm{m}^{2} \mathrm{~s}^{-1}\right), \\
& \mathrm{x}=\text { sentido do escoamento }(\mathrm{m}), \\
& \mathrm{i}_{\mathrm{i}}=\text { intensi dadeinstantânea deprecipitação }\left(\mathrm{m} \mathrm{s}^{-1}\right) \text { e } \\
& \mathrm{T}_{\mathrm{i}}=\text { taxa de infiltração da água no solo }\left(\mathrm{m} \mathrm{s}^{-1}\right) .
\end{aligned}
$$

A relação entre vazão e profundidade do escoamento é determinada pela equação

$$
q=\alpha h^{\beta}
$$

Os valores de $\alpha$ e $\beta$ são obtidos a partir da equação de Manning, utilizando as seguintes relações

$$
\begin{aligned}
& \alpha=\frac{\sqrt{\mathrm{S}_{\mathrm{O}}}}{\mathrm{n}} \\
& \beta=\frac{5}{3}
\end{aligned}
$$

em que 
$\mathrm{n}=$ coeficiente de rugosi dade do terreno $\left(\mathrm{s} \mathrm{m}^{-1 / 3}\right) \mathrm{e}$

$\mathrm{S}_{\mathrm{o}}=$ declividade do terreno na direção do escoamento $\left(\mathrm{m} \mathrm{m}^{-1}\right)$.

A profundidade de escoamento é transformada em vazão pela equação 2 . Esta equação é então substituída na equação 1 , sendoa equação resultante resolvida pelo método de di ferenças finitas, de acordo com al goritmo proposto por Braz (1990).

A intensidade instantânea de precipitação $\left(i_{i}\right)$ é obtida por meio da equação desenvolvida por Pruski et al. (2001), a qual é representada por

$$
i_{i}=i_{m}\left(1-\frac{c t}{t+b}\right)
$$

em que (mm),

$\mathrm{i}_{\mathrm{m}}=$ intensidade máxima média de precipitação

ceb $=$ parâmetros da equação de chuvas intensas (adm) e

$\mathrm{t}=$ duração da precipitação (min).

A obtenção de $i_{m}$ podeser realizada para diversas localidades brasileiras pelo software Plúvio 1.3(7).

Os hidrogramas resultantes das encostas são obtidos resolvendo a equação 1 para diferentes intervalos de tempo $(\Delta \mathrm{t})$. Para cada $\Delta \mathrm{t}$ o modelo determina a vazão correspondente.

\section{Modelagem do escoamento superficial no canal}

A modelagem do escoamento superficial no canal é realizada utilizando a equação 6, proposta por J ulien et al. (1995). Esta equação é obtida a partir do model o de ondas cinemáticas.

$$
\frac{\partial \mathrm{A}}{\partial \mathrm{t}}+\frac{\partial \mathrm{Q}}{\partial \mathrm{x}}=\mathrm{qr}_{\mathrm{r}}
$$

em que $\left(\mathrm{m}^{2}\right)$,

$A=$ seção transversal mol hada pelo escoamento

$\mathrm{Q}=$ vazão escoada no canal $\left(\mathrm{m}^{3} \mathrm{~s}^{-1}\right)$,

$\mathrm{q}_{\mathrm{r}}=$ vazão resultante por unidade de largura, proveniente do leito da estrada e da área externa de contribuição $\left(\mathrm{m}^{2} \mathrm{~s}^{-1}\right)$.

$$
q_{r}=q_{e}+q_{s c}
$$

em que

$\mathrm{q}_{\mathrm{e}}=$ vazão, por unidade de largura, proveniente do leito da estrada $\left(\mathrm{m}^{2} \mathrm{~s}^{-1}\right)$ e

$\mathrm{q}_{\mathrm{sc}}=$ vazão, por unidade de largura, proveniente da área externa de contribuição $\left(\mathrm{m}^{2} \mathrm{~s}^{-1}\right)$.

(7) Software para a obtenção dos parâmetros da equação de chuvas intensas para diferentes localidades do Brasil, podendo ser obtido em www.ufv.br/dea/gprh.
Para obter a vazão escoada no canal, a equação 6 foi resolvida pelo método de diferenças finitas, segundo o al goritmo proposto por Braz (1990), sendo a área de escoamento obtida por meio da equação

$$
\mathrm{Q}=\alpha_{c} \mathrm{~A}^{\beta_{\mathrm{c}}}
$$

em que $\alpha_{c}$ e $\beta_{c}$ foram obtidos para o escoamento no canal, considerando-se um rearranjo da equação de Manning, resultando, para canal triangular, nas equações

$$
\begin{gathered}
\alpha_{\mathrm{c}}=\frac{\sqrt{\mathrm{s}_{\mathrm{f}}}}{\mathrm{n}} \frac{\left(\mathrm{m}_{1}+\mathrm{m}_{2}\right)^{1 / 3}}{2^{1 / 3}\left(\sqrt{\mathrm{m}_{1}^{2}+1}+\sqrt{\mathrm{m}_{2}^{2}+1}\right)^{2 / 3}} \\
\beta_{\mathrm{c}}=\frac{4}{3}
\end{gathered}
$$

\section{Período de retorno para a determinação do hidrograma de escoamento}

O período de retorno utilizado para a determinação do hidrograma de escoamento superficial foi definido com base no período médio de duração de um leito encascalhado, conforme CEBTP (Centre Experimental de Recherches et D'etudes du Batiment et Travaux Publics), citado por Pastore (1997), o qual é de três anos.

\section{Determinação da tensão de cisalhamento provocada pelo escoamento}

A partir do momento em que é determinado o hidrograma de escoamento no canal da estrada, o modelo, para cada metro de canal, determina a tensão cisalhante associada ao escoamento, utilizando a equação da resistência proposta por Chow (1959). Tendo em vista quea tensão provocada pelo escoamento em canais não ocorre de maneira uniforme em toda a seção transversal, foi considerada apenas a tensão máxima, que provoca as maiores perdas de solo. Desta forma, o valor do raio hidráulico $\left(R_{h}\right)$ da equação da resistência foi substituído pela profundidade de escoamento, conforme a equação

$$
\tau_{\mathrm{E}}=\frac{\gamma \mathrm{y} S}{0,102}
$$

em que

$\tau_{\mathrm{E}}=$ tensão provocada pelo escoamento ( $\left.\mathrm{Pa}\right)$,

$\gamma=$ peso específico da água $\left(\mathrm{kg} \mathrm{m}^{-3}\right)$,

$\mathrm{y}=$ profundidade do escoamento $(\mathrm{m}) \mathrm{e}$

$\mathrm{S}=$ dedividade do canal $\left(\mathrm{m} \mathrm{m}^{-1}\right)$.

A profundidade de escoamento é determinada com base na vazão de escoamento, declividade do canal e nas suas características geométricas.

\section{Cálculo do espaçamento entre desagua- douros}

Para que não ocorra erosão no canal, a tensão provocada pelo escoamento deverá ser inferior ou, 
no máximo, igual àquela que o solo é capaz de resistir. Este critério, entretanto, é bastante rigoroso, uma vez que, em decorrência do desgaste provocado pel o próprio tráfego, existe a necessidade da realização de manutenções periódicas noleito da estrada e, desta forma, também nas suas margens, permitindo que pequenos danos provocados pela erosão nos canais possam ser facilmente recuperados. Outro aspecto a ser considerado está relacionado com o custo de instal ação do sistema de drenagem, o qual aumenta para condições nas quais as perdas sejam consideradas nulas. Tendo em vista estes aspectos, considerou-se um valor admissível para o aprofundamento do canal de drenagem em decorrência do processo erosivo, de tal forma que este seja atingido quando da realização da manutenção já prevista para a estrada.

\section{Aprofundamento máximo tolerável no canal de drenagem}

O aprofundamento máximo para o canal de drenagem da estrada $\left(a_{\mathrm{pm}}\right)$ deve ser atingido em intervalo de tempo igual ao período tomado para manutenção da estrada, o qual é o mesmo utilizado como período de retorno da precipitação para determinação do hidrograma. Sendo assim, o valor de $a_{\mathrm{pm}}$ corresponde a um val or que não comprometa o tráfego e que permita a apropriada manutenção da estrada.

\section{Perda de solo tolerável no canal}

$O$ volumedesolo $\left(V_{s}\right)$ a ser removido, considerando o conceito de $\mathrm{a}_{\mathrm{pm}}$, é determinado pela equação

$$
\mathrm{V}_{\mathrm{s}}=\mathrm{A} \mathrm{a}_{\mathrm{pm}}
$$

em que

$\mathrm{V}_{\mathrm{S}}=$ volume de solo a ser removido pela erosão $\left(\mathrm{cm}^{3}\right)$,

A = área da superfície do solo considerada para efeito de cálculo $\left(\mathrm{cm}^{2}\right)$ e

$a_{\mathrm{pm}}=$ aprofundamento máximo $(\mathrm{cm})$. $1 \mathrm{~cm}^{2}$.

A área A, para efeito de cálculo, foi fixada em
A perda de sol o tolerável $\left(P_{s t}, g\right)$ correspondente a $V_{S}$ é determinada pela equação

$$
\mathrm{P}_{\mathrm{st}}=\mathrm{V}_{\mathrm{s}} \rho_{\mathrm{s}}
$$

em que $\rho_{\mathrm{s}}$ é a massa específica do solo $\left(\mathrm{g} \mathrm{cm}^{-3}\right)$.

\section{Perda de solo provocada pelo escoamento}

A partir das características do canal e do escoamento superficial, a vazão foi transformada em profundidade de escoamento. Desta forma, utilizando a equação 11, o hidrograma de escoamento foi transformado em um gráfico de tensão cisalhante ao longo do tempo, obtendo-se, para cada intervalo de $1 \mathrm{~m}$ de canal, o perfil de variação da tensão provocada pelo escoamento ao longo do tempo. Na figura 2, encontra-se a representação gráfica da variação da tensão cisalhante ao longo do tempo, bem como a região considerada para o cálculo da perda de solo provocada pelo escoamento, sendo também ilustrados os valores do intervalo de tensão considerado e da tensão média referente a cada intervalo.

A partir do momento em que a tensão provocada pelo escoamento supera a tensão crítica de cisal hamento, ocorre o desprendimento e a conseqüente perda desolo, a qual é determinada pela equação

$$
\mathrm{PS}_{\mathrm{e}}=\sum_{\mathrm{t} 1}^{\mathrm{t} 2}\left[\left(\tau_{\mathrm{M}}-\tau_{\mathrm{c}}\right) \Delta \mathrm{tKA}\right]
$$

em que

$\mathrm{PS}_{\mathrm{e}}=$ perda de solo provocada pelo escoamento superficial $(g)$,

$\tau_{\mathrm{M}}=$ tensão média de cisalhamento durante $\mathrm{o}$ intervalo de tempo $\Delta \mathrm{t}(\mathrm{Pa})$,

$\tau_{\mathrm{c}}=$ tensão crítica de cisal hamento do solo $(\mathrm{Pa})$,

$\Delta \mathrm{t}=$ intervalo de tempo ( $\mathrm{min})$,

$\mathrm{K}$ = erodiblidade do solo $\left(\mathrm{g} \mathrm{cm}^{-2} \mathrm{~min}^{-1} \mathrm{~Pa}^{-1}\right) \mathrm{e}$

$\mathrm{A}=$ área da superfície do solo considerada para efeito de cálculo $\left(\mathrm{cm}^{2}\right)$.

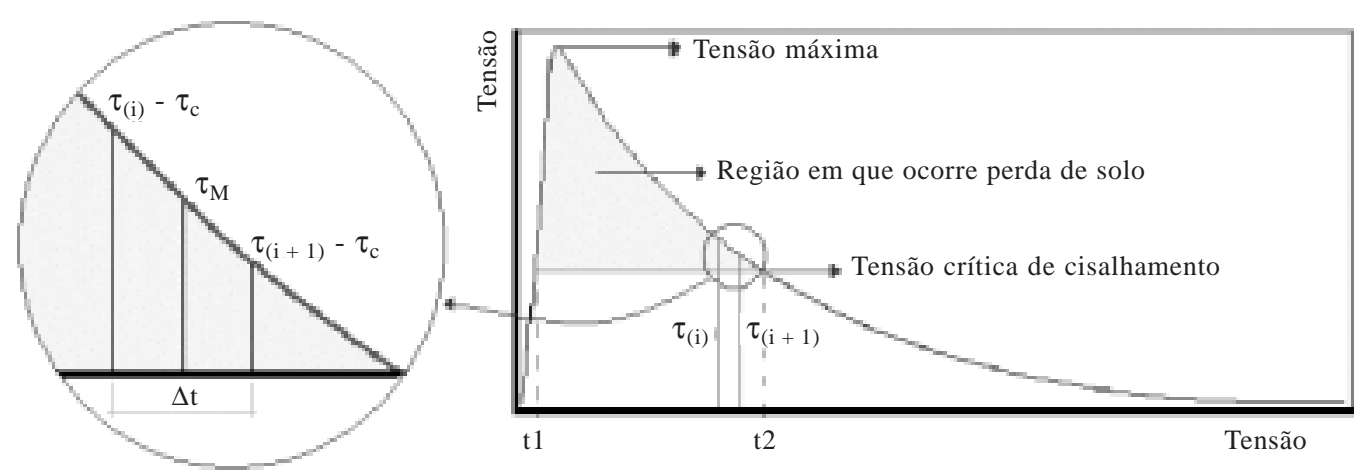

Figura 2. Representação da variação da tensão cisalhante com o tempo para o escoamento no canal de drenagem de uma estrada, indicando a região de interesse para a determinação da perda de solo provocada pelo escoamento, o intervalo de tempo $(\Delta t)$ e a tensão média referente a este intervalo $\left(\tau_{M}\right)$. 
O valor de $\tau_{M}$ é obtido pela equação

$$
\tau_{M}=\frac{\tau(\mathrm{i})+\tau(\mathrm{i}+1)}{2}
$$

em que

$\tau_{(\mathrm{i})}=$ tensão provocada pel o escoamento no tempo i (Pa) e,

$\tau_{(\mathrm{i}+1)}=$ tensão provocada pelo escoamento no tempo $\mathrm{i}+1(\mathrm{~Pa})$.

Valores de $\tau_{M}$ inferiores a $\tau_{c}$ significam que a tensão média provocada pelo escoamento no intervalo de tempo é inferior à tensão crítica, indicando que não ocorrerá perda de solo neste interval o de tempo.

O valor de $\Delta$ t é obtido pela equação

$$
\Delta \mathrm{t}=\mathrm{t}_{(\mathrm{i}+1)}-\mathrm{t}_{(\mathrm{i})}
$$

em que i e i +1 representam, respectivamente, os tempos correspondentes ao início e ao final do intervalo considerado.

\section{Espaçamento máximo recomendável entre desaguadouros}

A identificação do espaçamento recomendável entre desaguadouros foi feita pela comparação entre a perda provocada pelo escoamento e a perda tolerável, sendo real izada a cada metro de canal. O espaçamento máximo édeterminado quandoa perda provocada pelo escoamento supera a perda de solo tomada como tolerável, indicando, assim, a necessidade da existência de um desaguadouro.

\section{Análise do desempenho do método desenvolvido}

Nas simulações, foi utilizada a equação de chuvas intensas desenvolvida por Pinto (1995) para a localidade de Viçosa (MG), e considerado o período de retorno de três anos para a determinação do espaçamento entre desaguadouros:

$$
i_{m}=\frac{1082,798 T^{0,265}}{(t+23,781)^{0,775}}
$$

em que

$\mathrm{i}_{\mathrm{m}}=$ intensidade máxima média de precipitação $\left(\mathrm{mm} \mathrm{h}^{-1}\right)$,

$$
\begin{aligned}
& T=\text { período de retorno (anos) e } \\
& t=\text { duração da preci pitação (min). }
\end{aligned}
$$

Para identificar as áreas de contribuição, foi considerada uma estrada com semilargura de $3 \mathrm{~m}$, declividade transversal de $3 \%$ e taxa de infiltração de $1 \mathrm{~mm} \mathrm{~h}^{-1}$, com rugosidadehidráulica de $0,012 \mathrm{~s} \mathrm{~m}^{-1 / 3}$. Foi também considerada uma seção de corte com largura de $12 \mathrm{~m}$, declividade de $20 \%$ e taxa de infiltração de $20 \mathrm{~mm} \mathrm{~h}^{-1}$, sem cobertura e com rugosidade hidráulica de $0,10 \mathrm{~s} \mathrm{~m}^{-1 / 3}$. O canal da estrada foi tomado como triangular, com incl inação das paredes de 10/1 para o leito da estrada e de 1/1 para a seção de corte; a rugosidade hidráulica foi assumi da como 0,018 $\mathrm{s} \mathrm{m}^{-1 / 3}$. A massa específica do solo no canal foi considerada como $1,30 \mathrm{~g} \mathrm{~cm}^{-3}$.

Nas simulações realizadas, foram mantidas constantes todas as condições descritas anteriormente, sendo alteradas apenas as características referentes à variável em análise. As variáveis analisadas neste trabalho foram as seguintes: erodi bilidade e tensão crítica para cisalhamento do solo (Quadro 1), aprofundamento máximo do canal (5 e $10 \mathrm{~cm}$, correspondentes a perdas de solo de 6,5 e $13 \mathrm{~g}$, respectivamente, conforme a equação 13).

\section{RESULTADOS E DISCUSSÃO}

\section{Análise do modelo considerando diferentes tipos de solos}

Na figura 3, estão representados os resultados de perda de solo com base nos dados de resistência do solo apresentados no quadro 1 e para uma declividade de $5 \%$, sendo destacada a posição de aprofundamento máximo do canal da estrada de $5 \mathrm{~cm}\left[6,5 \mathrm{~g} \mathrm{~cm}^{-2}\right]$.

Para este aprofundamento os espaçamentos entre desaguadouros para os diferentes solos foram de

Quadro 1. Valores de erodi bilidade em sulcos e tensão crítica para cisalhamento do solo para diferentes solos em condições de estradas, conforme apresentados por Morfin et al. (1996)

Característica do leito

Característica do solo

Argiloso Siltoso Arenoso Argiloso encascalhado Arenoso encascalhado

\begin{tabular}{clllll}
\hline $\mathrm{K}^{(1)}$ & 0,0012 & 0,0036 & 0,0024 & 0,0018 & 0,0018 \\
$\tau_{\mathrm{c}}{ }^{(2)}$ & 1,5 & 1,8 & 2 & 1,8 & 2 \\
\hline${ }^{(1)}$ Erodibilidade do solo $\left(\mathrm{g} \mathrm{cm}^{-2} \mathrm{~min}^{-1} \mathrm{~Pa}^{-1}\right){ }^{(2)}$ Tensão crítica para cisalhamento do solo (Pa).
\end{tabular}

${ }^{(1)}$ Erodibilidade do solo $\left(\mathrm{g} \mathrm{cm}^{-2} \mathrm{~min}^{-1} \mathrm{~Pa}^{-1}\right) .{ }^{(2)}$ Tensão crítica para cisalhamento do solo (Pa). 


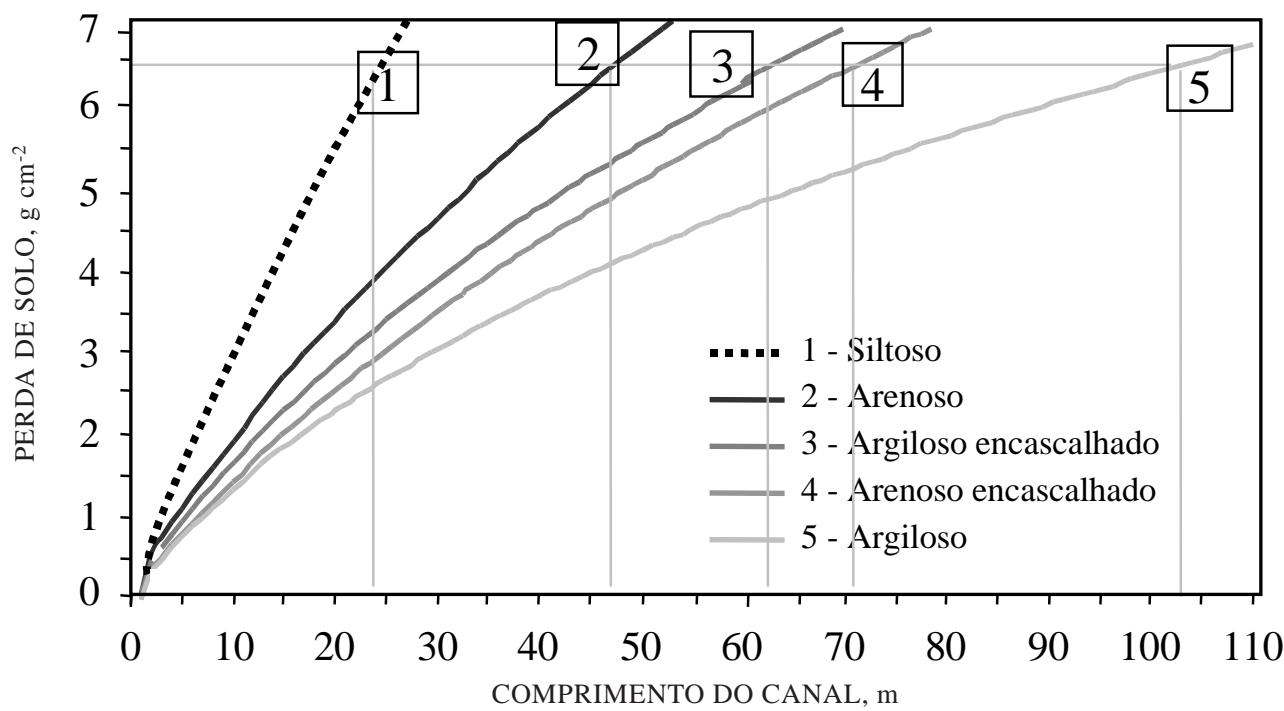

Figura 3. Perdas de solo na base do canal ao longo do seu comprimento, utilizando dados de erodibilidade e tensão crítica de cisalhamento para diferentes texturas de solo, apresentados por Morfin et al. (1996), para declividade de $5 \%$, destacando-se a perda de solo para $5 \mathrm{~cm}\left(6,5 \mathrm{~g} \mathrm{~cm}^{-2}\right)$ de aprofundamento de canal.

$103 \mathrm{~m}$ (argiloso), $71 \mathrm{~m}$ (arenoso encascalhado), $63 \mathrm{~m}$ (argiloso encascalhado), $47 \mathrm{~m}$ (arenoso) e $23 \mathrm{~m}$ (siltoso). Estes dados indicam que, para um mesmo aprofundamento máximo tolerável de canal, o solo argiloso proporcionou maior espaçamento entre desaguadour os ou, para um mesmo comprimento de canal, este solo apresentou menores perdas, indicando que este foi o mais resistente à erosão. Para o solo siltoso, o comportamento foi o oposto, indicando ser ele o menos resistente à erosão.

Tomando por base o sol o mais resistente à erosão (argil oso) e que, conseqüentemente, proporcionou o maior espaçamento, os demais espaçamentos representam, em relação a este: 68,9 \% (arenoso encascalhado), 61,2\% (argiloso encascalhado), 45,6 \% (arenoso) e 22,3 \% (siltoso).

O comportamento evidenciado corresponde ao esperado em condições reais, nas quais os solos mais resistentes, aqui representados por menores val ores de erodibilidade e maiores val ores de tensão crítica de cisalhamento, podem apresentar maiores espaçamentos entre desaguadouros. O menor valor obtido para o espaçamento entre desaguadouros (solo siltoso) é atribuído ao fato de a erodibilidade apresentada por este (Quadro 1) ser três vezes superior àquela apresentada pel o solo argil oso, o que resulta da menor coesão entre as partículas para aquel e sol o em relação ao solo argi loso, acarretando, conseqüentemente, menor resistência ao processo erosivo. O resultado obtido confere com o apresentado por Santos et al. (1988), os quais comentam que solos com maiores teores de argila são mais resistentes à erosão do que solos com el evado teor de silte.
A importância da erodibilidadee a sua influência no comprimento máximo determinado pel o modelo podem também ser visual izadas quando comparados os solos arenoso e arenoso encascalhado, os quais apresentam a mesma tensão crítica de cisal hamento, e o fato de o solo encascalhado apresentar menor erodibilidade faz com que o comprimento máximo admissível seja maior para este tipo de solo.

É possível, avaliar a variação da perda de solo ao Iongo do canal, bem como obter os espaçamentos recomendáveis entre desaguadouros, considerando diferentes limites de perdas desolo (Figura 3). Nesta figura, étambém possível evidenciar quea perda de sol o e, conseqüentemente, o aprofundamento do canal, apresentam taxa de variação mais acentuada nos menores comprimentos, sendo esta taxa reduzida à medida que o comprimento aumenta. Estefato é explicado pela variação na profundidade de escoamento ao longo do canal. Com o aumento da vazão, a profundidade de escoamento também aumenta; entretanto, no caso de canais triangulares (como o utilizado nas simulações), a taxa de aumento da profundidade de escoamento é decrescente com o aumento da vazão, uma vez que a largura da superfície aumenta. Assim sendo, para os menores comprimentos, os incrementos de vazão provocam maiores incrementos na profundidade de escoamento e, conseqüentemente, na tensão máxima de cisalhamento.

\section{Análise do modelo para diferentes valores de declividade do canal}

$\mathrm{Na}$ figura 4, são apresentados os espaçamentos obtidos, considerando declividades do canal de 5 e $10 \%$ e um aprofundamento máximo $\left(\mathrm{a}_{\mathrm{pm}}\right)$ de $5 \mathrm{~cm}$. 
Esta figura evidencia que o espaçamento máximo recomendável entre desaguadouros diminui expressivamente com o aumento da declividade do terreno, o que pode ser explicado pelo fato de a declividade do canal interferir, diretamente, como multiplicador, na equação para determinação da tensão de cisal hamento provocada pel o escoamento (Equação 11).

Embora o aumento da declividade tenha efeito também na profundidade do escoamento (y), uma vez que y é reduzida com o acréscimo na vel ocidade de escoamento, este efeito é muito menos expressivo queaqueleassociadoà influência direta da declividade (Equação 11); entretanto, faz com que a alteração provocada na declividade acarrete uma variação nãolinear no espaçamento entre desaguadouros.
Considerando os cinco solos analisados, os espaçamentos obtidos para a declividade de $10 \%$ foram, em média, $22 \%$ daqueles obtidos para a declividade de $5 \%$.

\section{Análise do modelo para diferentes valores de aprofundamento do canal}

$\mathrm{Na}$ figura 5, são apresentados os espaçamentos obtidos considerando valores de $a_{\text {pm }}$ de 5 e $10 \mathrm{~cm}$. Os val ores dos espaçamentos entre desaguadouros, quando do aumento no valor de $a_{\mathrm{pm}}$ de $5 \mathrm{~cm}\left(\mathrm{P}_{\mathrm{st}}=\right.$ $6,5 \mathrm{~g})$ para $10 \mathrm{~cm}\left(P_{\mathrm{st}}=13 \mathrm{~g}\right)$, foram de: $283,5 \%$ (argil oso), 211,3 \% (arenoso encascal hado), 220,6\% (argil oso encascal hado), 185,1 \% (arenoso) e 173,9 \% (siltoso). Evidenciou-se, portanto, que a variação do espaçamento obtido não foi linear com a variação

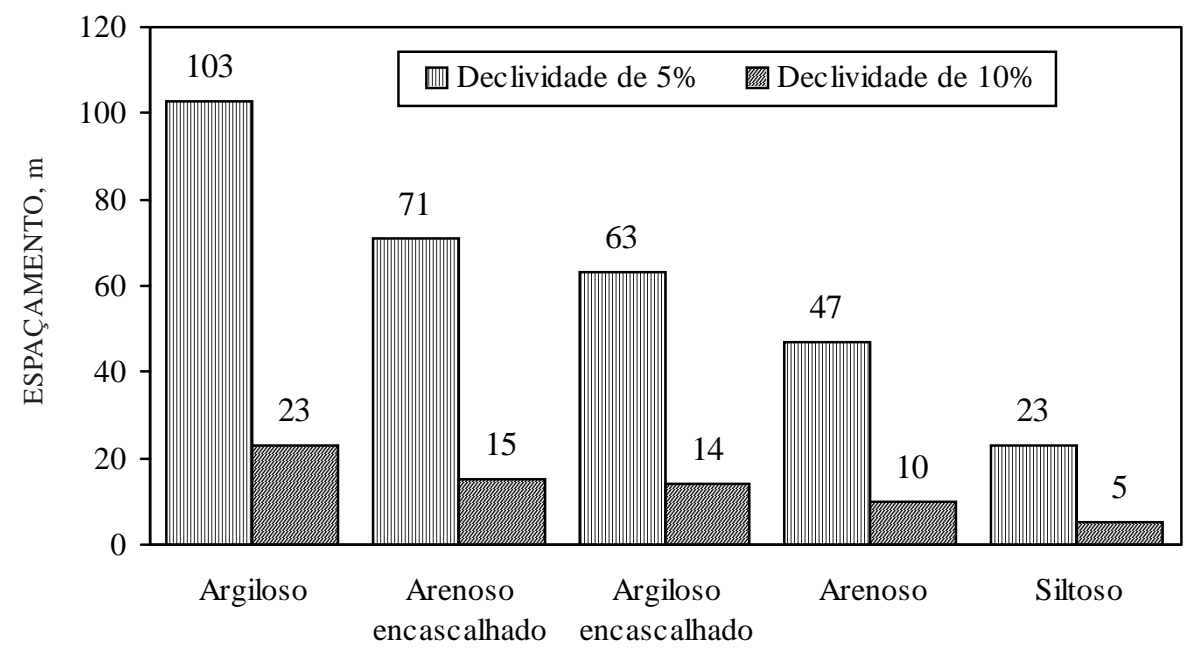

TIPO DE MATERIAL DO LEITO

Figura 4. Espaçamentos máximos entre desaguadouros obtidos utilizando dados de erodi bilidade e tensão crítica de cisalhamento para diferentes texturas de solo para declividades de 5 e $10 \%$ e $5 \mathrm{~cm}$ de aprofundamento do canal.

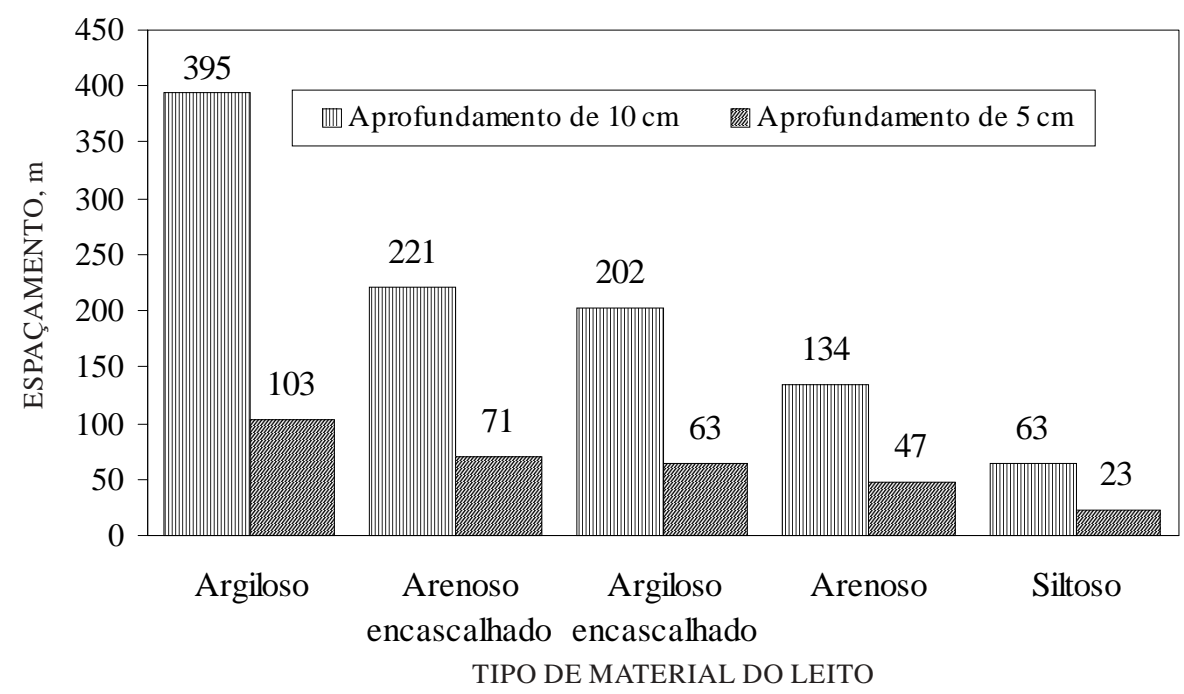

Figura 5. Espaçamentos máximos entre desaguadouros obtidos utilizando dados de erodibi lidadee tensão crítica de cisal hamento para diferentes texturas de solo para aprofundamentos do canal de 5 e $10 \mathrm{~cm}$ e declividade de $5 \%$ 
do aprofundamento tolerável, uma vez que este foi aumentado em $100 \%$ e os resultados superaram sensivel mente estevalor. Esta diferença decorre do fato de que, para canais triangulares, a variação da profundidade de escoamento e, conseqüentemente, da tensão cisal hante não é linear com o aumento no comprimento do canal, conforme já descrito anteriormente.

\section{CONCLUSÕES}

1. O model o desenvolvido permitiu determinar o espaçamento entre desaguadouros, considerando as condições do leito da estrada e das suas áreas de contribuição, da preci pitação do local analisado e da resistência do sol o ao cisalhamento.

2. O model o mostrou-se sensível às alterações na erodi bilidade e na tensão crítica de cisal hamento do solo, tendo o aumento da erodibilidade e a redução na tensão crítica de cisalhamento provocado reduções no espaçamento entre desaguadouros.

3. O aumento na declividade do canal da estrada reduziu o espaçamento entre desaguadouros.

4. O aumento do aprofundamento máximo tolerável para o canal proporcionou aumentos no espaçamento entre desaguadouros.

\section{LITE RATURA CITADA}

ANJ OS FILHO, O. Estradas de terra. J ornal O Estado de São Paulo, São Paulo. 29 deabril de 1998. (SuplementoAgrícola).

BERTOLINI, D.; DRUGOWICH, M. I.; LOMBARDI NETO, F. \& BELINAZZI J ÚNIOR, R. Controle deerosão em estradas rurais. Campinas, CATI, 1993. 37p. (Boletim Técnico, 207)

BRAZ, R.L. Hydrology - an introduction to hydrologic science. New York, Addison-Wesley Publishing Company, 1990. $643 p$.

BUBLITZ, U. \& CAMPOS, L.C. Adequação de estradas rurais em microbacias hidrográficas - especificações de projetos e serviços. Curitiba, EMATER-PR, 1992. 70p. (Boletim Técnico, 18)

CHOW, V.T. Open channel hydraulics. New York, McGrawHill Book Company, 1959. 680p.
DNER. Anuário estatístico dos transportes: GEIPOT, 2000. disponível em <www.dner.gov.br. $>$. acesso em Abril 2002.

GRACE III, J .M.; RUMMER, B.; STOKES, B.J . \& WILHOIT, J . Evaluation of erosion control techniques on forest roads. Trans. Am. Soc. Agric. Eng., 41:383-391, 1998.

J ULIEN, P.Y.; SAGHAFIAN, B. \& OGDEN, F.L. Raster-based hydrologic modeling of spatially-varied surface runoff. Water Res. Bull., 31:523-536, 1995.

MORFIN, S.; ELLIOT, W.; FOLTZ, R. \& MILLER, S. Predicting effects of climate, soil, and topography on road erosion with the WEPP model. In: ANNUAL INTERNATIONAL MEETING. Phoenix, 1996. Proceeding. Phoenix, American Society of Agriculture Engeenering. 1996.

MUÑOZ-CARREDA, R. \& PARSONS, J .E. VFMOD - Vegetative filter strips hydrology and sediment transport model: model documentation and user's manual, version 1.04. North Carolina, Biological and Agricultural Engineering- NCSU, 1999. 92p.

NOGAMI, J .S. \& VILLIBOR, D.F. Pavimentação de baixo custo com solos lateríticos. São Paulo, Villibor, 1995. 240p.

PASTORE, E. Estudos para planejamento da conservação de estradas deterra do município e de usinas de açúcar eálcool de São J oaquim da Barra - Estado de São Paulo. In: SIMPÓSIO INTERNACIONAL DE PAVIMENTAÇÃO DE RODOVIAS DE BAIXO VOLUME DE TRÁFEGO, 1., Rio de J aneiro, 1997. Anais. Rio de J aneiro, Associação Brasileira de Pavimentação, 1997. p.1-11.

PINTO, F.A. Chuvas intensas no Estado de Minas Gerais: análises emodel os. Viçosa, Universidade Federal deViçosa, 1995. 87p. (Tese de Doutorado)

PRUSKI, F.F.; GRIEBELER, N.P. \& SILVA, D.D. Comparação entre dois métodos para a determinação do volume de escoamento superficial. R. Bras. Ci. do Solo, 2:403-410, 2001.

REID, L.M. \& DUNNE, T. Sediment production from forest roads. Water Res. Res, 20:1753-1761, 1984.

SANTOS, A.R.; PASTORE, E.L.; AUGUSTO J ÚNIOR, F. \& CUNHA, M.A. Estradas vicinais de terra: Manual técnico para manutenção e conservação. São Paulo, Instituto de Pesquisas Tecnológicas, 1988. 125p. (Manual Técnico)

SILVA, J .M. Metodologia para obtenção do hidrograma de escoamento superficial ao longo de uma encosta. Viçosa, Universidade Federal de Viçosa, 1999. 64p. (Tese de Mestrado) 
Nori Paulo Griebeler et al.

R. Bras. Ci. Solo, 29:397-405, 2005 